\title{
Characterization of OSIRIS NAC filters for the interpretation of multispectral data of comet 67P/Churyumov-Gerasimenko
}

N. Oklay ${ }^{1}$, J.-B. Vincent ${ }^{1}$, H. Sierks ${ }^{1}$, S. Besse ${ }^{2}$, M. Pajola ${ }^{3}$, I. Bertini ${ }^{3}$, H. Rickman ${ }^{4,5}$, F. La Forgia ${ }^{6}$, A. M. Barucci ${ }^{7}$, S. Fornasier ${ }^{7,8}$, C. Barbieri ${ }^{3,6}$, D. Koschny ${ }^{2}$, P. L. Lamy ${ }^{9}$, R. Rodrigo ${ }^{10,11}$, J. Agarwal ${ }^{1}$, M. F. A'Hearn ${ }^{12}$, J.-L. Bertaux ${ }^{13}$, G. Cremonese ${ }^{14}$, V. Da Deppo ${ }^{15}$, B. Davidsson ${ }^{5}$, S. Debei ${ }^{16}$, M. De Cecco ${ }^{17}$, M. Fulle ${ }^{18}$, O. Groussin ${ }^{9}$, P. J. Gutiérrez ${ }^{19}$, C. Güttler ${ }^{1}$, S. F. Hviid ${ }^{20}$, W.-H. Ip ${ }^{21}$, L. Jorda ${ }^{9}$, H. U. Keller ${ }^{22}$, J. Knollenberg ${ }^{20}$, J.-R. Kramm ${ }^{1}$, E. Kührt ${ }^{20}$, M. Küppers ${ }^{23}$, L. M. Lara ${ }^{19}$, M. Lazzarin ${ }^{6}$, J. J. Lopez-Moreno ${ }^{19}$, F. Marzari' ${ }^{6}$, H. Michalik ${ }^{24}$, G. Naletto ${ }^{25,3,15}$, N. Thomas ${ }^{26}$, and C. Tubiana ${ }^{1}$

1 Max-Planck-Institut für Sonnensystemforschung, Justus-von-Liebig-Weg 3, 37077 Göttingen, Germany e-mail: oklay@mps.mpg.de

2 Research and Scientific Support Department, European Space Agency, 2201 Noordwijk, The Netherlands

3 Center of Studies and Activities for Space, CISAS, "G. Colombo", University of Padova, via Venezia 15, 35131 Padova, Italy

${ }^{4}$ PAS Space Research Center, Bartycka 18A 00-716 Warszawa, Poland

5 Department of Physics \& Astronomy, Uppsala University, Box 51675120 Uppsala, Sweden

6 Dipartimento di Fisica e Astronomia "Galileo Galilei”, University of Padova, vic. Osservatorio 3, 35122 Padova, Italy

7 LESIA, Observatoire de Paris, CNRS, UPMC Université Paris 06, Université Paris-Diderot, 5 place J. Janssen, 92195 Meudon Principal Cedex, France

${ }^{8}$ Université Paris-Diderot, Sorbonne Paris Cité, 4 rue Elsa Morante, 75205 Paris Cedex 13, France

9 Aix Marseille Université, CNRS, LAM (Laboratoire d'Astro-physique de Marseille) UMR 7326, 13388 Marseille, France

10 Centro de Astrobiologia (INTA-CSIC), European Space Agency (ESA), European Space Astronomy Centre (ESAC), PO Box 78, 28691 Villanueva de la Canada, Madrid, Spain

11 International Space Science Institute, Hallerstrasse 6, 3012 Bern, Switzerland

12 Department for Astronomy, University of Maryland, College Park, MD 20742-2421, USA

13 LATMOS, CNRS/UVSQ/IPSL, 11 boulevard d'Alembert, 78280 Guyancourt, France

14 INAF Osservatorio Astronomico di Padova, vic. dell'Osservatorio 5, 35122 Padova, Italy

15 CNR-IFN UOS Padova LUXOR, via Trasea 7, 35131 Padova, Italy

16 Department of Industrial Engineering, University of Padova, via Venezia 1, 35131 Padova, Italy

17 University of Trento, via Sommarive 9, 38123 Trento, Italy

18 INAF Osservatorio Astronomico di Trieste, via Tiepolo 11, 34014 Trieste, Italy

19 Instituto de Astrofisica de Andalucia-CSIC, Glorieta de la Astronomia, 18008 Granada, Spain

20 Deutsches Zentrum für Luft- und Raumfahrt (DLR), Institut für Planetenforschung, Rutherfordstraße 2, 12489 Berlin, Germany

${ }^{21}$ Graduate Institute of Astronomy, National Central University, 300 Chung-Da Rd, 32054 Chung-Li, Taiwan

22 Institut für Geophysik und extraterrestrische Physik, TU Braunschweig, 38106 Braunschweig, Germany

23 Operations Department European Space Astronomy Centre ESA, PO Box 78, 28691 Villanueva de la Cañada, Madrid, Spain

24 Institut für Datentechnik und Kommunikationsnetze der TU Braunschweig, Hans-Sommer-Str. 66, 38106 Braunschweig, Germany

25 Department of Information Engineering, University of Padova, via Gradenigo 6/B, 35131 Padova, Italy

26 Physikalisches Institut, Sidlerstrasse 5, University of Bern, 3012 Bern, Switzerland

Received 28 February 2015 / Accepted 29 August 2015

\section{ABSTRACT}

Context. We interpret multicolor data from OSIRIS NAC for the remote-sensing exploration of comet 67P/Churyumov-Gerasimenko. Aims. We determine the most meaningful definition of color maps for the characterization of surface variegation with filters available on OSIRIS NAC.

Methods. We analyzed laboratory spectra of selected minerals and olivine-pyroxene mixtures seen through OSIRIS NAC filters, with spectral methods existing in the literature: reflectance ratios, minimum band wavelength, spectral slopes, band tilt, band curvature, and visible tilt.

Results. We emphasize the importance of reflectance ratios and particularly the relation of visible tilt vs. band tilt. This technique provides a reliable diagnostic of the presence of silicates. Color maps constructed by red-green-blue colors defined with the green, orange, red, IR, and $\mathrm{Fe}_{2} \mathrm{O}_{3}$ filters let us define regions that may significantly differ in composition.

Key words. comets: individual: 67P/Churyumov-Gerasimenko - methods: data analysis - techniques: imaging spectroscopy

\footnotetext{
* Appendices are available in electronic form at http://www. aanda.org
} 


\section{Introduction}

The OSIRIS scientific imager (Optical, Spectroscopic, and Infrared Remote Imaging System, Keller et al. 2007) onboard ESA's spacecraft Rosetta is an instrument designed to map the comet nucleus of 67P/Churyumov-Gerasimenko (67P) with high spatial resolution, at best a few centimeters per pixel. It provides color information on the surface and studies gas emission in the coma through a set of 26 dedicated filters. OSIRIS will contribute to the definition of the comet terrains, lithology, and mineralogy in synergy with the other instruments onboard the Rosetta spacecraft. This is a common approach in space missions and has proven to be very effective in several occasions. Color maps of many bodies have been produced and analyzed, for instance, the HRI camera on Deep Impact imaged both comets 9P/Tempel 1 (Sunshine et al. 2006) and 103P/Hartley 2 (Li et al. 2013), and the Framing Camera on Dawn revealed important color differences on the surface of asteroid (4) Vesta (Reddy et al. 2012).

The OSIRIS cameras observe comet 67P in the wavelength range of 250-1000 $\mathrm{nm}$ with a combination of 12 filters for the narrow angle camera (NAC) and in the wavelength range of 240-720 $\mathrm{nm}$ with a combination of 14 filters for the wide angle camera (WAC). NAC filters are designed for surface composition studies, while WAC filters were selected for gas coma emission studies. This study focuses on a set of techniques targeting the surface composition, that is, which minerals and compositional information can be derived from the NAC filters.

Detection and separation of the minerals was very important early on in the mission, during the global mapping phase (August-September 2014), because the NAC filter selection could be modified for future observations according to the observed spectral features. At 3.7 AU from the Sun, comet 67P was already active in this phase. However, most of the activity was concentrated in the Hapi region (the transition area between the two lobes of the comet), while other regions were far less active, or not at all (Sierks et al. 2015; Thomas et al. 2015). This trend is compatible with what we expected from earlier ground-based observations, which showed that the active fraction of the surface is quite limited (Snodgrass et al. 2013; Combi et al. 2012) and is no more than $4 \%$ of the total area.

To understand the compositional and morphologic changes induced by activity, it is therefore very important to map the dormant surface in high resolution and update these maps regularly to characterize the evolution of the surface. Observations of resurfaced terrains will lead to understanding the deeper layers and provide hints about the previous surface before activity started. Additionally, by comparing the current mineralogy with the formation conditions of the various minerals, we can reconstruct some of the chemical history of the comet.

We now describe how we derived the composition and mineralogy of the surface from our observations. The NAC filters (Table 1) were selected for this purpose, but mineralogic identification is not necessarily unique when only a few bandpasses are available in the spectrum. Therefore we discuss here the best filter combinations and analysis techniques for unambiguously identifying different minerals with the set of filters available on OSIRIS NAC.

\section{Minerals on comets}

Comets are believed to be formed by primordial dusty ice material during the formation of the early solar system (Weidenschilling 2004). Their dust is a mixture of silicates, both
Table 1. Filters of the narrow angle camera (adopted from Keller et al. 2007).

\begin{tabular}{cccc}
\hline \hline & $\begin{array}{c}\text { Wavelength } \\
(\mathrm{nm})\end{array}$ & $\begin{array}{c}\text { Bandwidth } \\
(\mathrm{nm})\end{array}$ & Science objective \\
\hline Far-UV & 269.3 & 53.6 & Surface spectral reflectance \\
Near-UV & 360.0 & 51.1 & Surface spectral reflectance \\
Blue & 480.7 & 74.9 & Surface spectral reflectance \\
Green & 535.7 & 62.4 & Surface spectral reflectance \\
Neutral & 640.0 & 520.0 & Neutral density filter \\
Orange & 649.2 & 84.5 & Surface spectral reflectance \\
Hydra & 701.2 & 22.1 & Water of hydration band \\
Red & 743.7 & 64.1 & Surface spectral reflectance \\
Ortho & 805.3 & 40.5 & Orthopyroxene \\
Near-IR & 882.1 & 65.9 & Surface spectral reflectance \\
$\mathrm{Fe}_{2} \mathrm{O}_{3}$ & 931.9 & 34.9 & Iron-bearing minerals \\
IR & 989.3 & 38.2 & IR surface reflectance \\
\hline
\end{tabular}

Notes. As a result of the limited spectral range of the laboratory data, the far-UV filter was excluded from this study. The neutral density filter is a broadband filter designed to reduce photon flux from bright sources and was not used for our spectral analysis.

amorphous and crystalline, Fe-Ni sulfides, small amounts of oxides, and other minerals. The most common cometary silicates are the $\mathrm{Mg}$-rich olivine (forsterite, $\mathrm{Fo}: \mathrm{Mg}_{2} \mathrm{SiO}_{4}$ ) and $\mathrm{Mg}$-rich pyroxene (enstatite, En: $\mathrm{Mg}_{2} \mathrm{Si}_{2} \mathrm{O}_{6}$ ) (Hanner \& Zolensky 2010).

In general, different silicate species form at significantly different temperatures. Direct condensation is a natural explanation for the abundance of Mg-silicates in comet dust. Forsterite and enstatite are predicted from thermodynamic models to be the first to condense in hot gas at $1200-1400 \mathrm{~K}$ and only react with iron at lower temperature. Therefore, they are assumed to have occurred in the hot inner solar nebula (Hanner 2003).

The most detailed information of the surface mineralogy of a comet has been obtained by analyzing the samples from comet 81P/Wild 2, collected during Stardust mission. The best-studied tracks mostly contain olivine, pyroxene, and sulfides. Olivine and pyroxene have a wide composition range with a peak at Fo99 and $\mathrm{En}_{95}$, respectively (Zolensky et al. 2006).

Observation of the Deep Impact ejecta by Spitzer revealed that olivine and pyroxene were the main components on comet 9P/Tempel 1 (Lisse et al. 2006). Comet 73P/SchwassmannWachmann 3 (Sitko et al. 2011) has also been observed with Spitzer, and the analysis showed that dust of both the 73P/SW3-B and 73P/SW3-C components is dominated by amorphous carbon and amorphous silicates of near-pyroxene composition. Comet C/1995 O1 Hale-Bopp shows Mg-rich olivines and pyroxenes according to the analysis of the obtained spectra both from ground- and space-based telescopes (Wooden et al. 1999). After the missions Vega 1, Vega 2, and Giotto, the dust composition of comet $1 \mathrm{P} /$ Halley was studied by many authors (see Hanner \& Zolensky 2010, for references) and the dominance of Mg-rich silicates is reported by Langevin et al. (1987). The common minerals found in these studies are Mg-rich silicates, which are the basis of our analysis. More detailed information on the astro-mineralogy can be found in Henning (2010).

The minerals listed in Table 2 were selected from the list of detected components in the samples of comet 81P/Wild 2 (Zolensky et al. 2006), collected during Stardust mission, and the calibration materials of the COmetary Secondary Ion Mass Analyzer (COSIMA) and the Grain Impact Analyser and Dust Accumulator (GIADA) instruments onboard Rosetta spacecraft (M. Hilchenbach; C. Engrand and A. Rotundi, priv. comm.). 
Table 2. Selected minerals.

\begin{tabular}{lllc}
\hline \hline & Minerals & & Number of data \\
\hline Anhydrous silicates & Olivine: & Forsterite & 23 \\
& & Fayalite & 15 \\
& Pyroxene: & Enstatite & 35 \\
& & Diopside & 13 \\
& & Fassaite & 4 \\
& Melilite & & 9 \\
Hydrated silicates & Serpentine & & 12 \\
& Smectite & 10 \\
Oxides & Ilmenite & 33 \\
& Magnetite & 7 \\
& Chromite & 13 \\
Carbonates & Corumdum & 1 \\
\multirow{2}{*}{ Sulfides } & Calcite & 18 \\
& Dolomite & 16 \\
& Pyrrhotite & 4 \\
\hline
\end{tabular}

Notes. Selected data have mixed sizes. See Appendix B for the details of the data used here.

Table 3. Mixtures (grain size $<45 \mu \mathrm{m}$ ).

\begin{tabular}{ccc}
\hline \hline Set number & HOSERLab ID & Mixture \\
\hline 1 & OCMIX1001 & OLV $(100 \%)$ \\
2 & OCMIX1002 & OLV $(90 \%)+$ PXY $(10 \%)$ \\
3 & OCMIX1003 & OLV $(80 \%)+$ PXY $(20 \%)$ \\
4 & OCMIX1004 & OLV $(70 \%)+$ PXY $(30 \%)$ \\
5 & OCMIX1005 & OLV $(60 \%)+$ PXY $(40 \%)$ \\
6 & OCMIX1006 & OLV $(50 \%)+$ PXY $(50 \%)$ \\
7 & OCMIX1007 & OLV $(40 \%)+$ PXY $(60 \%)$ \\
8 & OCMIX1008 & OLV $(30 \%)+$ PXY $(70 \%)$ \\
9 & OCMIX1009 & OLV $(20 \%)+$ PXY $(80 \%)$ \\
10 & OCMIX1010 & OLV $(10 \%)+$ PXY $(90 \%)$ \\
11 & OCMIX1011 & PYX $(100 \%)$ \\
\hline
\end{tabular}

Laboratory data of these selected minerals were collected from the publicly available database Reflectance Experiment LABoratory (RELAB) ${ }^{1}$. RELAB files of the selected minerals given in Table 2 are listed in Table B.1. Some of these laboratory spectra have various samples in various sizes, which have various spectral resolutions. No chemical analysis of almost all selected samples is currently available. This prevents investigating the effect of the compositional differences of the samples on the spectral methods we use. The differences in grain size and composition of the samples cause the diversity in the scatter plots presented in Sect. 3. The iron contents, which are higher than expected in cometary silicates, would increase the spectral slopes (see Sect. 3.3) and band tilts (see Sect. 3.2) to a currently unknown degree. Therefore, the sample names given in Table 2 do not indicate the pure minerals, but the sample names given in the RELAB database with the file names given in Table B.1. The olivine and pyroxene mixtures listed in Table 3 are olivine OLV003 and pyroxene PYX150 from the Hyperspectral Optical Sensing for Extraterrestrial Reconnaissance Laboratory (HOSERLab) database ${ }^{2}$. The absolute spectra of these selected minerals observed by OSIRIS NAC filters were then calculated (see Sect. 3) for further analysis.

The list of mineral samples includes end-members of olivine (forsterite and fayalite), some pyroxenes including enstatite,

\footnotetext{
1 http://www.planetary . brown.edu/relabdocs/relab.htm

2 http://psf.uwinnipeg.ca/Home.html
}

which is the most common pyroxene found in comets, two hydrated silicates so that we can detect water or hydration, and some expected mineral oxides, carbonates, and sulfides. The main focus of this study are the silicates, preferably the most commonly found silicates in comets, and hydrated silicates. As tracers of water, the hydrated silicates are particularly important and can be detected through their characteristic bands at $700 \mathrm{~nm}$ and $3000 \mathrm{~nm}$. OSIRIS is only sensitive to the $700 \mathrm{~nm}$ band, but Vilas (1994) and other studies (see Rivkin et al. 2002 for a review) have shown a correlation of up to $80 \%$ between the two bands. Therefore an OSIRIS detection of the $700 \mathrm{~nm}$ band very likely is a diagnostic of hydrated minerals. Of course, this assumes that we have observations with additional filters next to the minima. A cross comparison with data from the Visible and InfraRed Thermal Imaging Spectrometer (VIRTIS) onboard the Rosetta spacecraft will help to confirm the detection.

While we here focus on the detection of abundant minerals, comets also contain volatiles. Cometary ices are more challenging for OSIRIS because their spectra do not have features that are directly detectable by OSIRIS in the visible wavelength range. Nonetheless, their presence can be inferred from a bluer slope in the visible spectrum. While this by itself is not a diagnostic, it has been interpreted as signature of ice deposits after imaging and spectroscopic observations of comets 9P/Tempel 1 (Sunshine et al. 2006) and 103P/Hartley 2 (Li et al. 2013). The Hapi region of comet $67 \mathrm{P}$, whose spectrum is bluer spectrum than the average cometary surface, was also the most active region of the comet (Sierks et al. 2015; Fornasier et al. 2015), and VIRTIS results (changes in the spectral slope and absorption feature width, Capaccioni et al. 2015) suggest small amounts of water ice in the active areas. No large icy patches have been observed so far, but Pommerol et al. (2015) presented exposure of water ice at the surface of boulders as the most plausible scenario to explain the bright features on the surface of comet 67P. Definite proof of volatiles on the surface of the comet will require very careful calibration and a more detailed analysis of the data and will be presented in future studies. Additionally, surface composition studies will require the characterization of the coma in between the comet surface and the camera.

\section{Spectral analysis methods used to separate minerals}

To determine methods for the mineralogical characterization of the comet surface, we tested several spectral parameters found in the literature, starting from the analysis presented by Le Corre et al. (2011), who used them to distinguish howardite, eucrite, and diogenite (HED) minerals on Vesta terrains observed by the Dawn framing camera filters. The authors proposed specific spectral methods (reflectance ratios, band minima wavelength, and spectral slopes) that could distinguish HEDs and color information to be used to identify Vesta's terrains. We followed their approach in characterizing minerals with the OSIRIS NAC filters and extend it by additionally studying band tilt, visible tilt, and band curvature techniques.

\subsection{Direct detection}

Before introducing the spectral techniques we used to detect and separate the selected minerals, we investigated whether some spectral features of the minerals could be identified directly in our NAC spectra. We limited the scope of the analysis to minerals that are most likely to be found on the surface of comet 67P, given the data obtained by other missions (Table 2). 

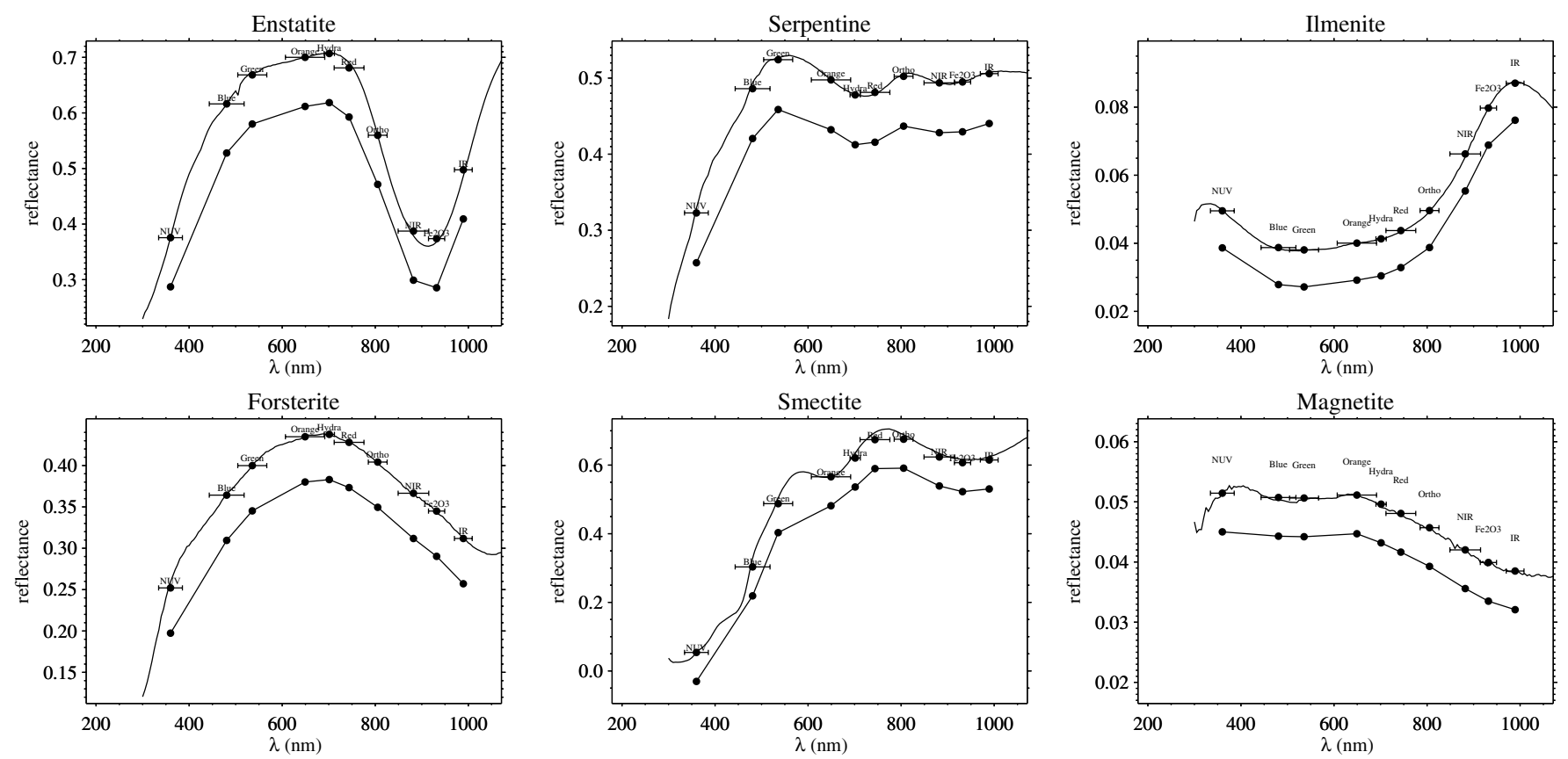

Fig. 1. Absolute spectra of anhydrous silicates (enstatite, forsterite) in the left panel, hydrated silicates (serpentine, smectite) in the middle panel, and oxides (ilmenite, magnetite) in the right panel as they would be observed through the full set of OSIRIS NAC filters. The solid lines above are laboratory data of the corresponding mineral with the central wavelengths of the NAC filters (dots) including their bandwidths (horizontal bars), while resampled laboratory data are arbitrarily (lowered by $1 / 8$ compared to the maxima) offset for display.

For each selected mineral, we resampled laboratory data to the OSIRIS spectral sensitivity by dividing the calibrated laboratory signal by the integrated area of the OSIRIS filter transmission curve over the same wavelength range. The same resampling was also applied to mixture spectra.

Figure 1 shows a few example spectra of various minerals as seen through NAC filters. These are the most common cometary silicates (Fig. 1, left panel), two hydrated silicates (Fig. 1, middle panel), and two oxides with bands around $500 \mathrm{~nm}$ (Fig. 1, right panel). More example spectra of the minerals presented in Table 2 are available in Appendix A (Fig. A.1). The $900 \mathrm{~nm}$ band in enstatite samples and the band around $1100 \mathrm{~nm}$ in forsterite samples shows that these samples are not pure and contain iron.

The resampled spectra in Fig. 1 demonstrate that OSIRIS NAC can detect the $900 \mathrm{~nm}$ band of enstatite and use this particular band to distinguish enstatite from other minerals. As a result of our limited spectral resolution, the $650 \mathrm{~nm}$ band of diopside will appear as a step in the spectrum as well. This is also true for the $700 \mathrm{~nm}$ and $900 \mathrm{~nm}$ bands of fassaite. More mineral examples are given in Fig. A.1 and are summarized here:

- the $900 \mathrm{~nm}$ bands of selected silicates are detected,

- the $700 \mathrm{~nm}$ band of serpentine is detected,

- sulfides have featureless spectra, but can be separated from other minerals by their slopes (Sect. 3.3),

- in the carbonate group, calcite has a very flat spectra, while dolomite resembles olivines,

- for oxides, the $500 \mathrm{~nm}$ band of ilmenite and magnetite will be detectable,

- chromite is difficult to distinguish from sulfides, while corundum is much more easily detectable.

Direct detection of chromite, corundum, calcite, troilite, and pyrrhotite will not be possible using OSIRIS NAC, but spectral techniques will help to separate them from each other and from other investigated minerals. The spectral range of our filters, which are limited to the visible and the near-infrared, does not allow us to observe the $1050 \mathrm{~nm}$ band of olivine, and the low spectral resolution prevents us from precisely measuring typical band parameters such as band depth or band areas of the detected bands. This apparent limitation, however, does not prevent OSIRIS from being able to separate different minerals, even when no bands are detected. We used various analytical techniques to parameterize the spectra in terms of reflectance ratios, spectral slopes, minimum band wavelength, and band curvature (measurable even if the band is not fully detected).

\subsection{Reflectance ratios}

Reflectance ratios describe the ratio of two filter values from the resampled spectra. According to the shape of the mineral spectrum or the existing features, these ratios can be used to distinguish different minerals. The main diagnostic here is the ratio of IR and $\mathrm{Fe}_{2} \mathrm{O}_{3}$ filters, which allows us to separate enstatite, serpentine, and smectite from the other listed minerals through the existing $900 \mathrm{~nm}$ band (see top left panel of Fig. 1). Reflectance ratios were combined with other spectral methods for the analysis.

Plotting the values obtained from one ratio against another one yields a two-dimensional map of these mineral parameters, in which different minerals will cluster at different locations.

Silicates are investigated with red/IR ratios, and IR/ $\mathrm{Fe}_{2} \mathrm{O}_{3}$ ratios show (Fig. 2) a suitable separation of enstatite from the other minerals with only three NAC filters (red, IR, and $\mathrm{Fe}_{2} \mathrm{O}_{3}$ ). Enstatite and forsterite (the two most abundant minerals detected on comets) are clearly separated with these ratios. Plotting all silicates together in Fig. 2 shows a few groups: diopside, enstatite, forsterite, fayalite, and fassaite are easily detected, while other silicates mostly fall onto the same place in the scatter plot. It is difficult to separate end-members of olivines (fayalite and forsterite) from each other with these combinations for the low red/IR ratios, but the separation is clear for higher ratios. 


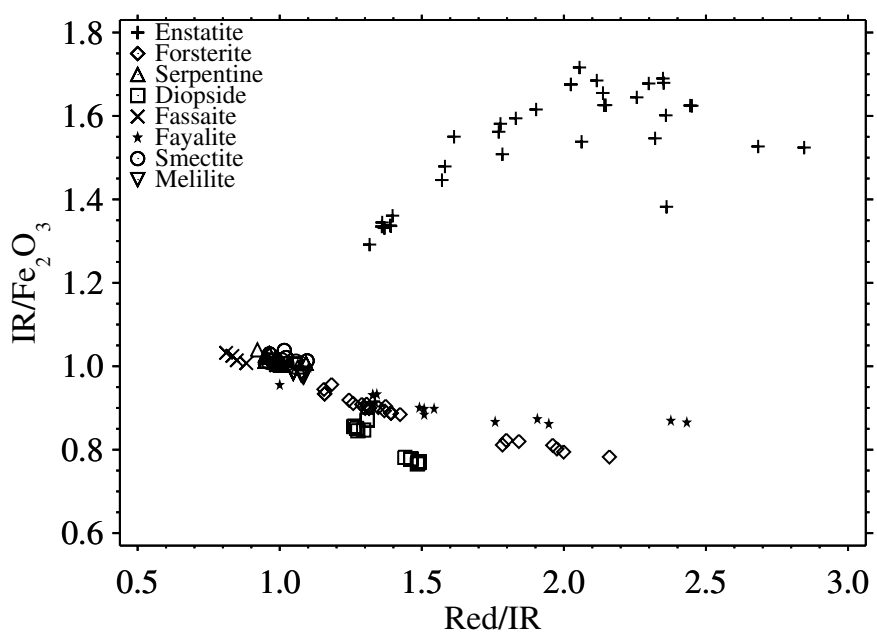

Fig. 2. Filter ratios of selected silicates.

Serpentine, smectite, and melilite are very difficult to separate from each other with these selected ratios. The fassaite and diopside groups are well separated with this ratio combination, as is enstatite. Olivine and pyroxene are separated from hydrated silicates. Hydrated silicates fall together as a group, but can be subdivided into smaller clusters. Ratio plots of IR/ $/ \mathrm{Fe}_{2} \mathrm{O}_{3}$ vs. green/orange better separate the most common cometary silicates (En, Fo) and hydrated silicates, as shown in the right panel of Fig. 9.

We define two particularly useful ratios:

1. The band tilt is the ratio between $\mathrm{Fe}_{2} \mathrm{O}_{3}$ and IR. It is used to separate enstatite, serpentine, and smectite through their detectable $900 \mathrm{~nm}$ band.

2. The visible tilt is the ratio between the blue and red filters. It is used to detect bluer patches on the comet surface, which are often associated with ice deposits (Sunshine et al. 2006; Li et al. 2013).

The top panel of Fig. 3 shows how band tilt and visible tilt are used to investigate silicates. The two-dimensional parameter map shows that enstatite always has a band tilt parameter lower than 0.8 , while other minerals have a band tilt of about 1.0 for smectite, serpentine, melilite, and fassaite and a tilt stronger than 1.0 for the remaining studied minerals. Enstatite, fayalite, diopside, forsterite and smectite are well separated in this plot, while other minerals cluster together. In this cluster only high visible-tilt values correspond to serpentine.

In the bottom panel of Fig. 3, the most common cometary silicates are plotted together with dolomite since the spectral shape of dolomite is similar to silicates because it includes ferrous ion, and it is investigated in visible tilt vs. band tilt plots. There are clearly two main clusters, enstatite alone, and forsterite and dolomite together. High visible-tilt and band-tilt values indicate forsterite, while lower values of the two quantities indicate dolomite.

The band tilt parameter is sensitive to the band intensity, which is sensitive to the actual mineral compositions. Because we lack a chemical analysis of the samples we used, the magnitudes of the groups are poorly constrained. However, the patterns in the clustering are reliable. This is valid for the analysis of the spectral slopes presented in the following section.
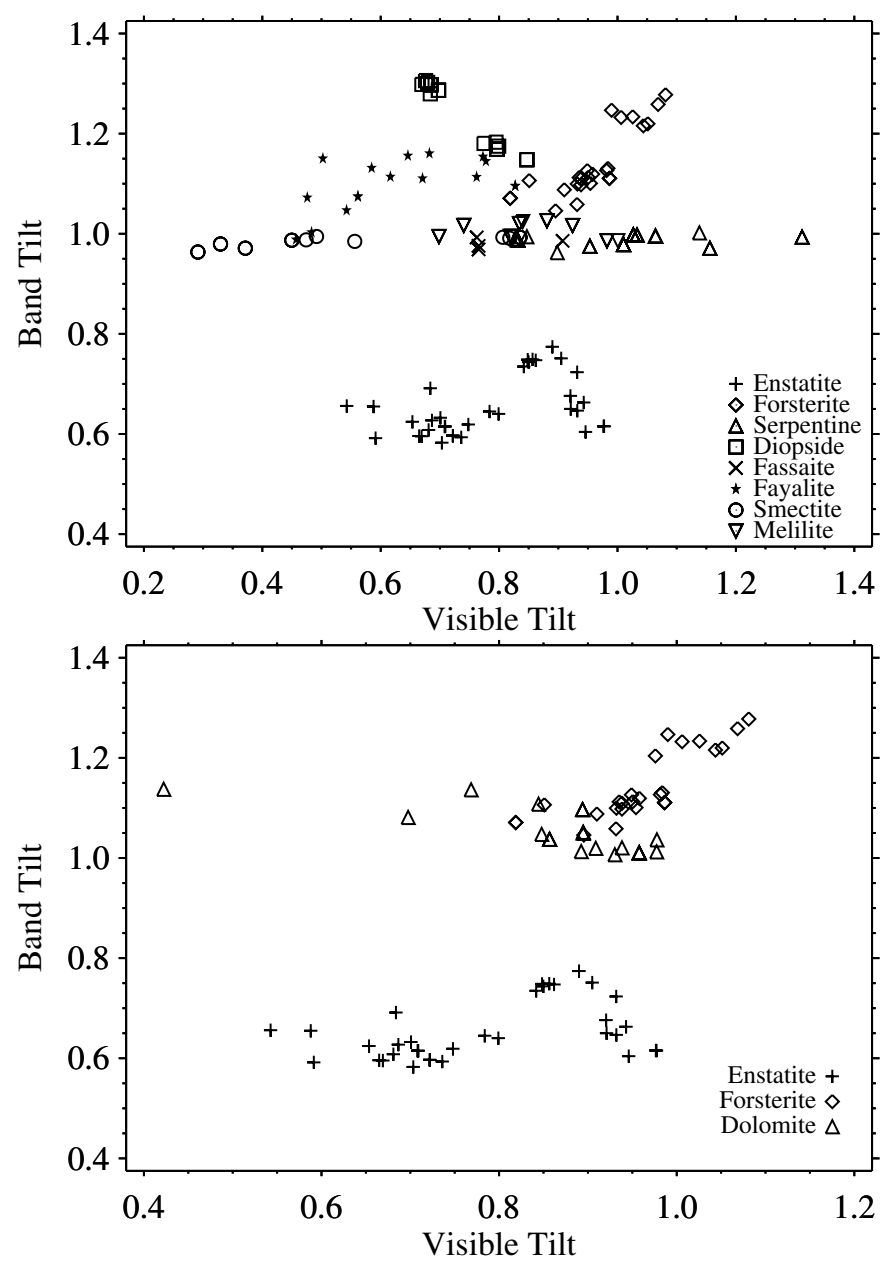

Fig. 3. Separation of minerals using visible tilt and band tilt.

\subsection{Spectral slopes}

The second technique we investigated is measuring an analytical spectral slope between red and $\mathrm{Fe}_{2} \mathrm{O}_{3}$ filters for silicates and carbonates, green-orange filters for oxides, and blue-red filters for sulfides. The slopes were then combined with reflectance ratios to separate the minerals.

The spectral slope is defined as spectral slope $=\arctan \left(\left(R_{2}-R_{1}\right) /\left(\lambda_{2}-\lambda_{1}\right)\right)$, where $R_{1,2}$ are the reflectance values in investigated filters and $\lambda_{1,2}$ are the corresponding central wavelengths.

We investigated the spectral slopes of silicates between the red and $\mathrm{Fe}_{2} \mathrm{O}_{3}$ filters together with the band-tilt values. The top panel of Fig. 4 shows the spectral slopes applied to all silicates. With the spectral slopes it is difficult to separate end-members of olivine from each other, but low-slope high band-tilt values only correspond to forsterite. Since the band minima of olivine occurs at longer wavelengths, its slope is shallower, and this results in the separation of enstatite from forsterite and fayalite. Enstatite, diopside, fassaite, smectite, and forsterite are well separated from the rest. The most common anyhdrous silicates (enstatite and forsterite) are plotted together with the hydrated silicates serpentine and smectite in the bottom panel of Fig. 4 versus the ratios of the green and Hydra filters. Hydrated silicates are better separated by this method. We also investigated the separation of these four silicates with various filter combinations. The best separation was achieved with the green and Hydra and the red and $\mathrm{Fe}_{2} \mathrm{O}_{3}$ filters (see Sect. 4 for more details). 

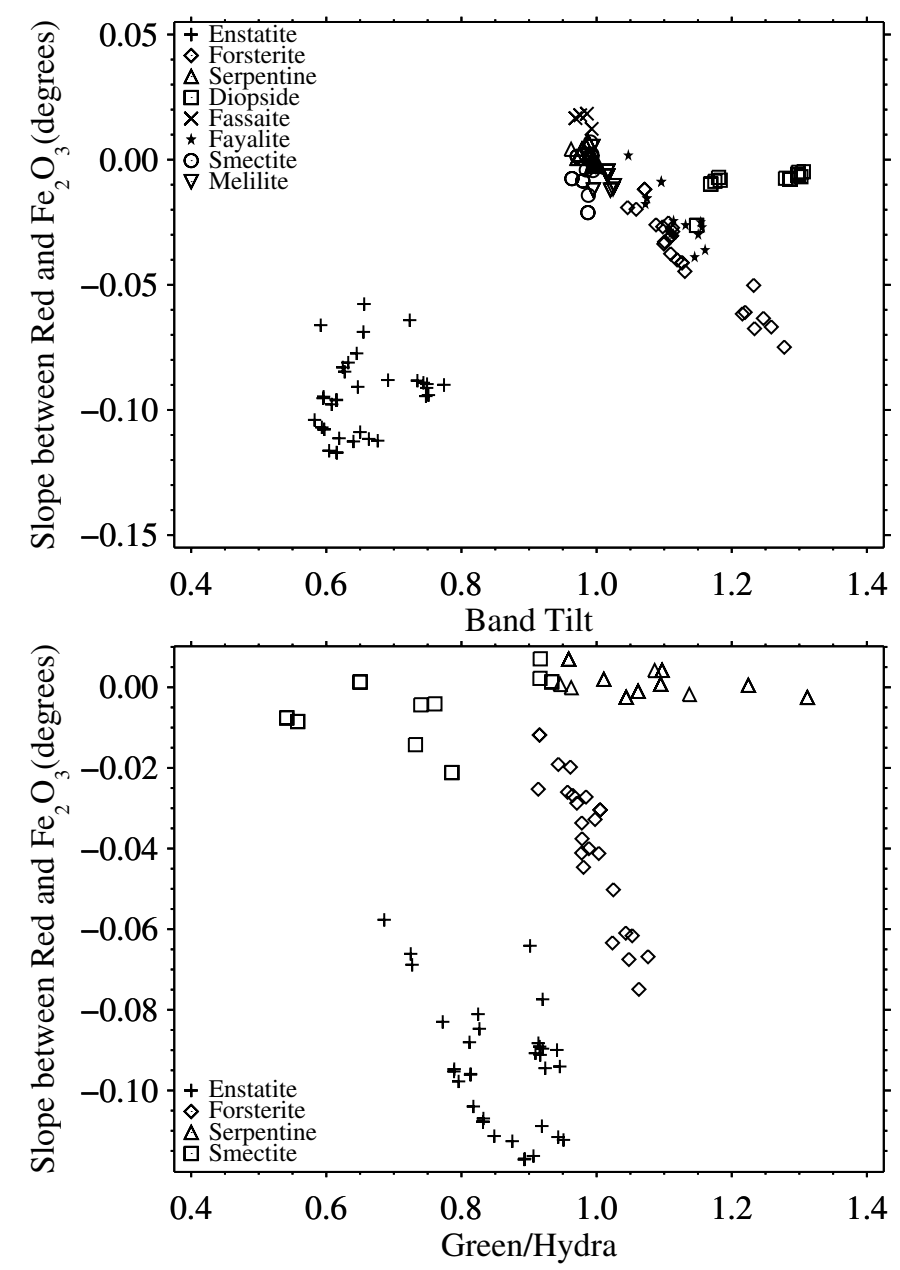

Fig. 4. Separation of silicates: slopes between red and $\mathrm{Fe}_{2} \mathrm{O}_{3}$ filters (top); slopes between green and Hydra filters (bottom).

The spectral slopes between the green and orange filters were used together with the ratios of near-UV and IR filters to investigate sulfides, which only have slope spectra in the observable range of the NAC. High ratios correspond to troilite, while low ratio high slope values correspond to pyrrhotite (top left panel of Fig. 5). Oxides can be separated with the slope between the blue and red filters against their band tilts (top right panel of Fig. 5). The slopes between the red and $\mathrm{Fe}_{2} \mathrm{O}_{3}$ filters were used together with the band tilt to investigate carbonates. The bottom left panel of Fig. 5 shows that the calcite band tilt is at unity and the spectral slope between the red and $\mathrm{Fe}_{2} \mathrm{O}_{3}$ filters is flat, while the band tilts and spectral slopes of dolomite vary more strongly and extend toward a higher band tilt and negative slopes. The large scatter of dolomite might be due to the compositional differences, as investigated in Gaffey (1986). From investigating minerals showing slope spectra in the NAC wavelength range, we found that it is more difficult to separate sulfides from oxides. Corundum, for which we have only one data set, falls far from the rest, and chromite and troilite are well separated from each other (bottom right panel of Fig. 5).

Because the spectra of dolomite are similar to those of enstatite and forsterite, as described in Sect. 3.2, they were investigated together and are presented in Fig. 6. These three minerals are presented with their spectral slopes calculated between the red and $\mathrm{Fe}_{2} \mathrm{O}_{3}$ filters and are plotted with band tilts. Even though dolomite is difficult to distinguish from forsterite for low slope values, the separation is clear in the high slope and high band-tilt values.

\subsection{Minimum band wavelength}

If an absorption band, or part of it, was detectable, we applied third-degree polynomial fits to derive the minimum band wavelength precisely. This is the case, for instance, in the range of ortho-NIR- $\mathrm{Fe}_{2} \mathrm{O}_{3}$-IR for the $900 \mathrm{~nm}$ bands of enstatite, serpentine, smectite, and the range of NUV-blue-green-orange for the $500 \mathrm{~nm}$ band of ilmenite and magnetite.

Figure 7 shows an example of measured band minima of different minerals, plotted against filter ratios. The minerals with bands are grouped together and investigated according to the location of their bands. The top panel of Fig. 7 shows that magnetite and ilmenite can be detected with a minimum band wavelength when it is combined with the ratios of the orange and NIR filters. The minimum band wavelength of magnetite is generally higher than that of ilmenite. Enstatite, serpentine, and smectite can be identified by the ratios of the orange and ortho filters (bottom panel of Fig. 7). The minimum band wavelengths of serpentine and smectite vary similarly, and the serpentine ratios are accumulated in unity, while lower ratios correspond to smectite data. Enstatite has a stable minimum band wavelength in the laboratory samples, but the selected ratio shows the variation in the spectra.

\subsection{Band curvature}

The band curvature is designed as diagnostic of the changes in the absorption feature at $900 \mathrm{~nm}$ and $500 \mathrm{~nm}$. The band curvatures are defined as follows:

Band curvature $= \begin{cases}\left(R_{805.3}+R_{989.3}\right) / R_{931.9} & \text { for the } 900 \mathrm{~nm} \text { band } \\ \left(R_{360.0}+R_{649.2}\right) / R_{480.7} & \text { for the } 500 \mathrm{~nm} \text { band, }\end{cases}$

where $R$ is the reflectance value at the indicated wavelength as subscript.

This method was then combined with the reflectance ratios and visible tilts.

Figure 8 shows the results of the band curvature method applied to the $900 \mathrm{~nm}$ bands of enstatite, serpentine, and smectite (bottom panel) and to the $500 \mathrm{~nm}$ bands of ilmenite and magnetite (top panel). The separation of the selected silicates is clear from the bottom panel of Fig. 8, while it is vaguer for oxides (top panel of Fig. 8). The high band curvatures shown in the bottom panel of Fig. 8 indicate enstatite, while low curvature values indicate hydrated silicates. The separation of the hydrated silicates was achieved with visible tilts. The top panel of the Fig. 8 shows that the high band curvatures indicate ilmenite, while low values indicate magnetite. Additionally, magnetite has only high ratios, while ilmenite is more diverse in wider range of ratios.

\section{Results}

Section 3 presented the various spectral techniques we used. Each of them is particularly suited for a given purpose, and not all of them are applicable to each selected minerals. For instance, band curvature and minimum band wavelength can only be applied to minerals that display an absorption band that is clearly resolved by our filters, that is, at $900 \mathrm{~nm}$ (enstatite, serpentine, and smectite) or $500 \mathrm{~nm}$ (ilmenite and magnetite). Spectral slopes are also more suitable for the minerals whose spectra do not show strong band or no spectral features in the NAC spectral 

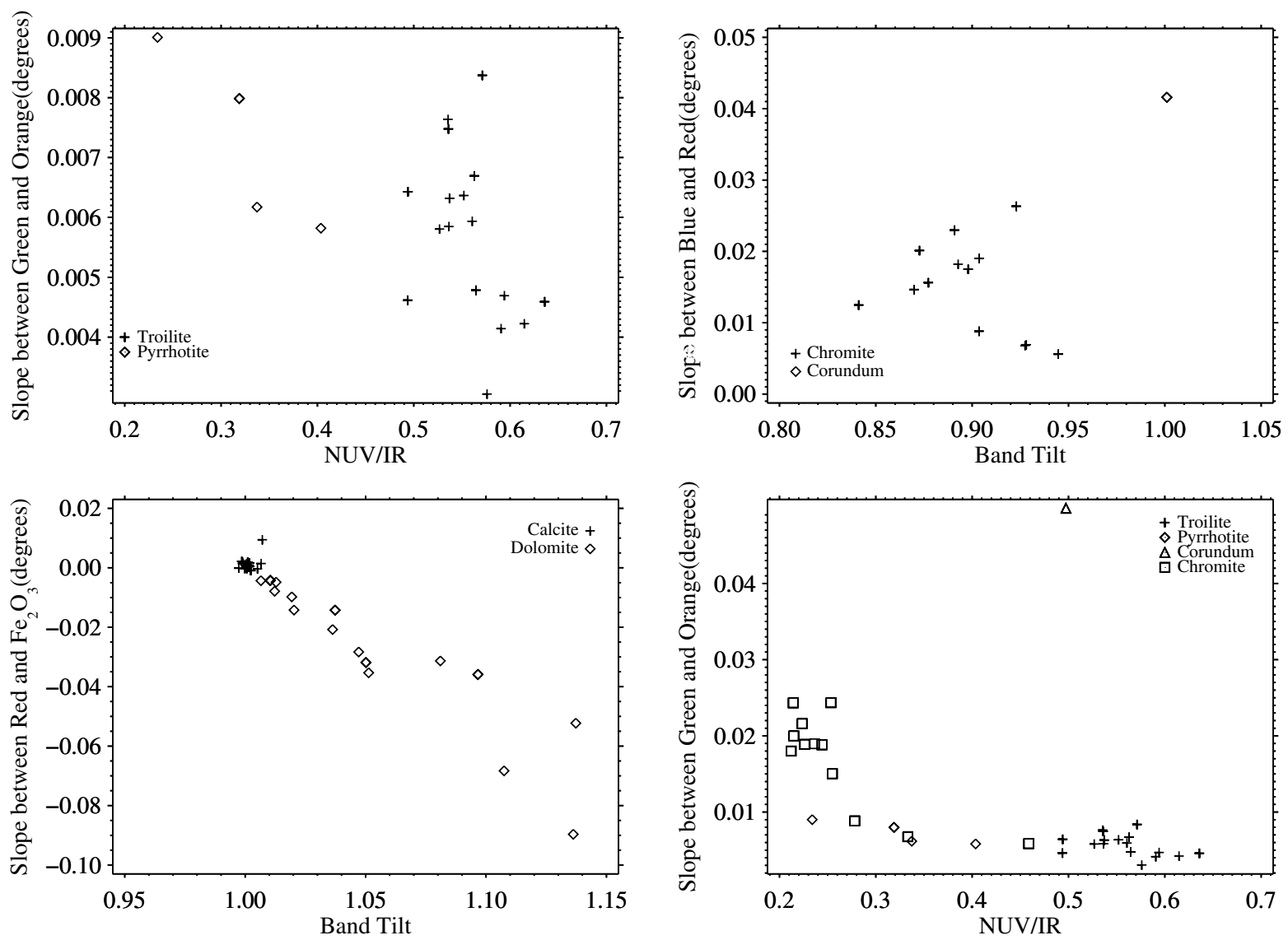

Fig. 5. Slopes vs. ratios for the separation of minerals without significant spectral features.

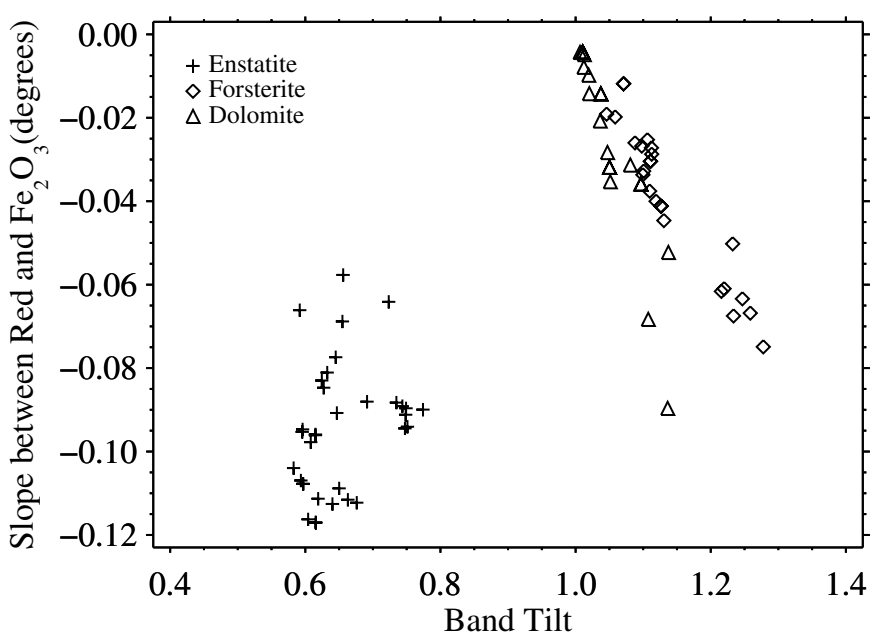

Fig. 6. Separation of minerals: slopes between the red and $\mathrm{Fe}_{2} \mathrm{O}_{3}$ filters.

range (corundum, chromite, troilite, and pyrrhotite). The band tilt is designed for the $900 \mathrm{~nm}$ band, therefore it is assumed to better fit the separation of enstatite, serpentine, and smectite from the other minerals. It appears that even when the absorption bands are poorly resolved with the OSIRIS NAC filters, we can still combine these techniques to obtain an accurate separation of various minerals.

Spectral techniques implying the usage of band parameters (minimum band wavelength and band curvature) are unsuitable for the separation of ilmenite and magnetite (top panels of Figs. 7 and 8). In this case, separation was achieved more by using the spectral ratios. The minimum band wavelength and band curvature methods work better for the silicates (bottom panels of Figs. 7 and 8) and separates the investigated silicates.

In general, we easily separated the most common minerals found in comets with the parameters defined in Sect. 3. A convenient way to represent this information is to assign each filter ratio to a given color and build a color map of the nucleus surface where each shade of red, green, and blue will correspond to a given mineralogy.

To detect silicates, we propose to use the red-green-blue (RGB) scale with $\mathrm{R}=\mathrm{red} / \mathrm{IR}, \mathrm{G}=$ green/orange, $\mathrm{B}=\mathrm{IR} / \mathrm{Fe}_{2} \mathrm{O}_{3}$. Each color range covers the strongest difference in all filter ratios. Figure 9 shows the parameter plots and separation of minerals using these definitions.

Figure 10 shows where each of the minerals falls on the RGB color model. For instance, enstatite lies in the red-magenta color space because it as much higher values in the ratios associated with red and blue than it does with green. On the other hand, serpentine has similar filter ratios in all colors and therefore is displayed as close to white in our color model.

Of course, the real material on the comet is likely to be made of mixtures of various materials. While studying the volatiles and organics is beyond the scope of this paper, it is useful to consider how a mixture of minerals would affect our analysis. Spectral changes with various mixing of olivine and pyroxene are represented in Fig. A.2 for the sampled laboratory data. An increasing pyroxene amount in the mixture results in a significantly flat spectrum. The main spectral difference occurs between $40 \%$ olivine and $30 \%$ olivine mixtures. The spectral techniques we defined to investigate the most common cometary silicates were applied to the mixtures. In the defined RGB ratios, 

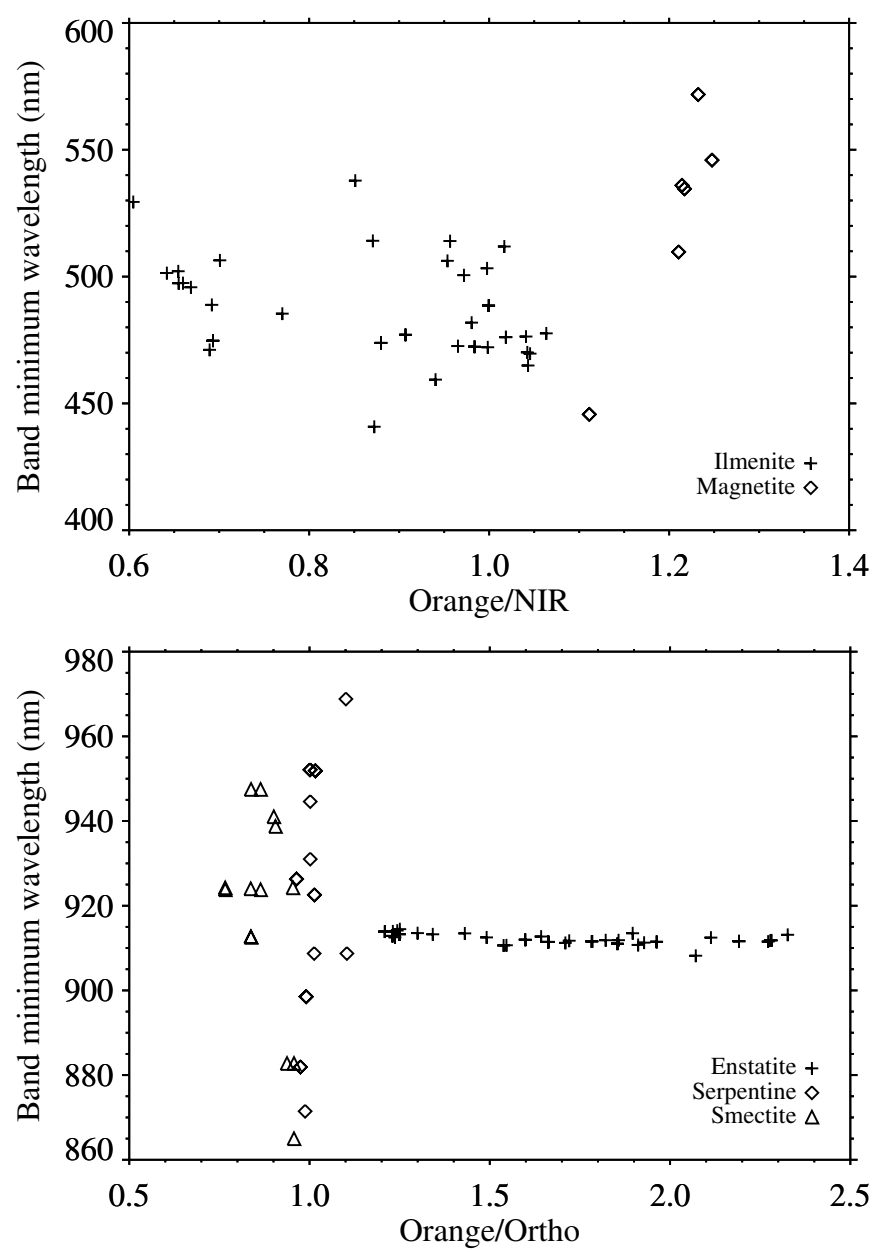

Fig. 7. Separation of oxides by their minimum band wavelength around $500 \mathrm{~nm}$ (top panel) and silicated by their minimum band wavelength around $900 \mathrm{~nm}$.

the mixtures with olivine from $100 \%$ to $40 \%$ fall on forsterite (the magnesium-rich end-member of olivine), and the mixtures with olivine from $30 \%$ to $100 \%$ pyroxene fall on serpentine clusters (Fig. A.3). These results obtained using the mixtures determine the limitation of our spectral methods. Even if forsterite and serpentine clusters might be hosting the olivine-pyroxene mixtures, the separation of smectite and enstatite from these two clusters will remain unchanged.

\section{Conclusions and outlook}

With all the methods presented here, we are able to distinguish enstatite samples from all other minerals. The detection of hydrated silicates and the most common cometary silicates requires at least observations with red, $\mathrm{Fe}_{2} \mathrm{O}_{3}$, and IR filters, but for color maps it is necessary to have observations in green and orange filters as well. Color maps produced by green, orange, red, IR, and $\mathrm{Fe}_{2} \mathrm{O}_{3}$ filters would allow us to detect hydrated silicates and the most common cometary silicates. In the maps created with the presented RGB strategy (Fig. 10), red will indicate pyroxenerich areas, while olivine-rich areas will be green, and hydrated silicates will be in blue tones. In the nominal observing case due to operational limitations, visible tilt vs. band tilt plots will be helpful to distinguish these silicates. The best separation of these four silicates is achieved by visible tilt vs. band tilt plots and by $\mathrm{IR} / \mathrm{Fe}_{2} \mathrm{O}_{3}$ vs. green/orange plots.

OSIRIS NAC will contribute to the detection of surface changes, either topographic or compositional. Our filter analysis
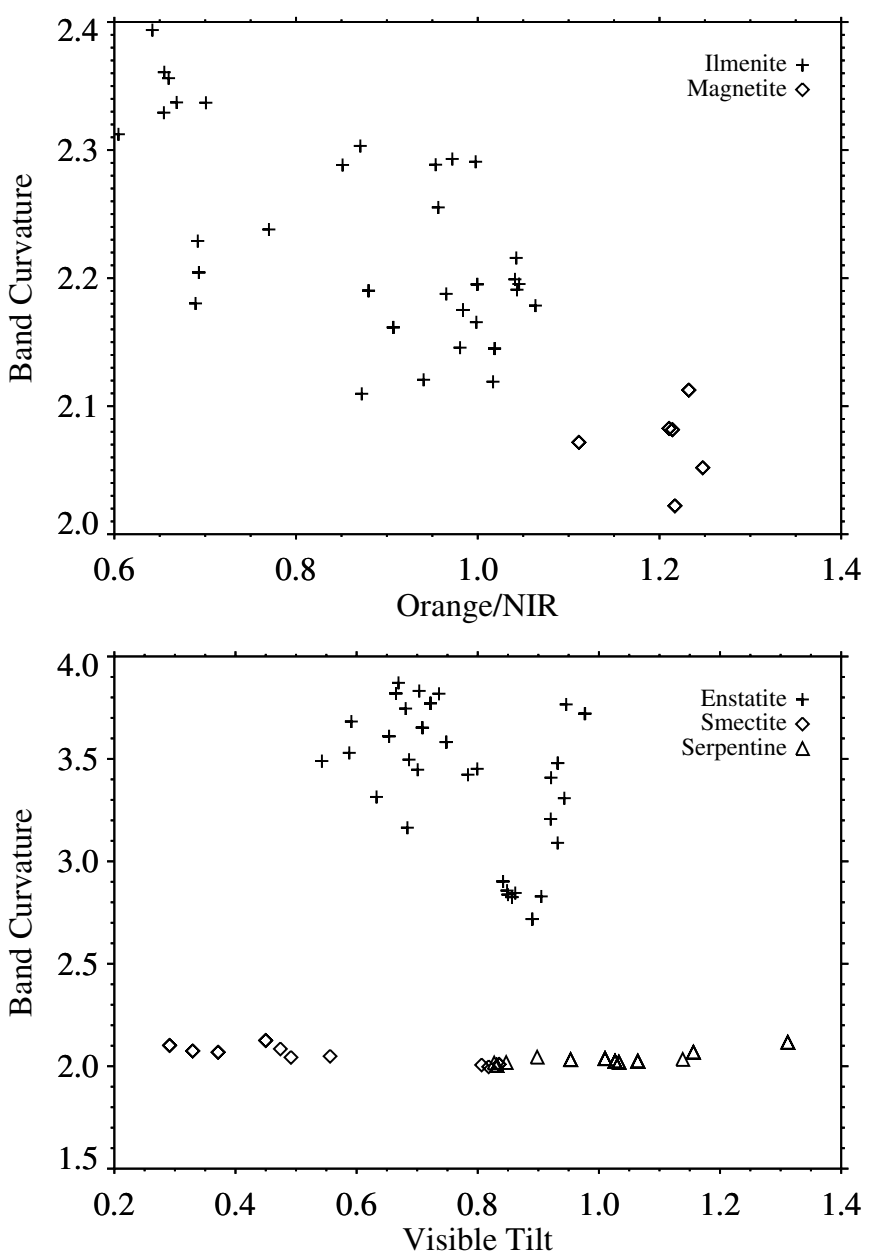

Fig. 8. Separation of minerals according to band curvature. The top panel shows the band curvature for the 0.9 micron band, while the bottom panel shows that for the 0.5 micron band.

is used to optimize the observing conditions and requirements on the detection of color variations on the surface.

Since each mineral forms in different initial conditions and after different processes, detecting them will place constraints on the history of the comet and the environment it experienced.

Our results are summarized in Table 4, and Table 5 shows the minimum observational requirements to detect some mineral bands in the observing range of NAC.

Our techniques are reliable enough for a first evaluation of local compositional differences. They will be improved with cross-calibration from measurements taken by the lander Philae, which will provide a ground-truth of what the surface is really made of. Similarly, synergistic observations with other remotesensing instruments will allow us to separate icy and mineral patches, and we will be able to define new diagnostics that are sensitive to the observed ices. Combining the high spatial resolution of OSIRIS NAC observations and the high spectral resolution of VIRTIS in the infrared range will help us to refine the detection of icy patches and study the relation of the $700 \mathrm{~nm}$ and $3000 \mathrm{~nm}$ bands.

With the observations and measurements made during the Rosetta mission, we will be able to compare composition results with those of Stardust and Deep Impact. The synergy among the Rosetta instruments will allow us to perform multidisciplinary science so that we can achieve the best possible understanding of comets. 

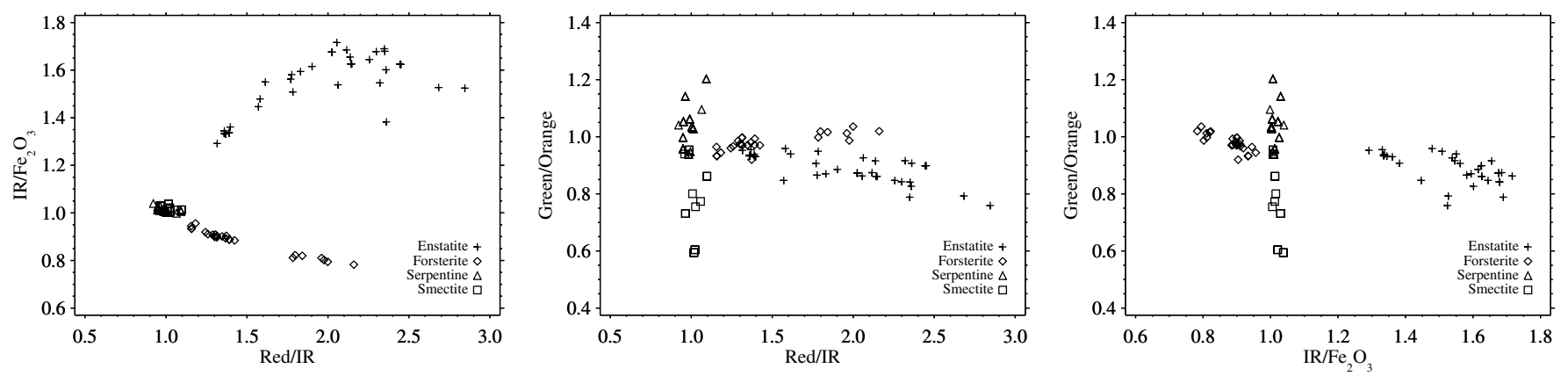

Fig. 9. Example of the three ratios used to define the $\mathrm{RGB}$ colors in the map: $\mathrm{R}=$ red/IR, $\mathrm{G}=$ green/orange, $\mathrm{B}=\mathrm{IR} / \mathrm{Fe}_{2} \mathrm{O}_{3}$.

Table 4. Proposed methods for detecting cometary minerals, listing required filters for the detection of spectral bands of silicates (700 and $900 \mathrm{~nm}$ ) and oxides $(500 \mathrm{~nm})$.

\begin{tabular}{cc}
\hline \hline Hydrated and anhydrous silicates & $\begin{array}{c}\text { green/orange vs. IR/Fe } / \mathrm{Fe}_{3} \\
\text { band tilt vs. slopes }\left(\mathrm{red}-\mathrm{Fe}_{2} \mathrm{O}_{3}\right) \\
\text { green/Hydra vs. slopes }\left(\mathrm{red} / \mathrm{Fe}_{2} \mathrm{O}_{3}\right) \\
\text { band tilt vs. visible tilt }\end{array}$ \\
\hline Enstatite-serpentine-smectite & $\begin{array}{c}\text { orange/ortho vs. min. band wl. } \\
\text { visible tilt vs. band curvature }\end{array}$ \\
\hline Ilmenite-magnetite & orange/NIR vs. min. band wl. \\
& orange/NIR vs. band Curvature \\
Chromite-corundum & band tilt vs. slopes (blue-red) \\
Sulfades & NUV/IR vs. slopes (green-orange) \\
Carbonades & band tilt vs. slopes (red-Fe $\mathrm{O}_{3}$ ) \\
\hline
\end{tabular}

Table 5. Minimum observational requirements for detecting cometary minerals.

\begin{tabular}{ccc}
\hline \hline Bands & Minerals & Filters \\
\hline 0.9 micron & enstatite, fassaite, smectite, serpentine & Hydra-NIR- $\mathrm{Fe}_{2} \mathrm{O}_{3}-\mathrm{IR}$ \\
0.7 micron & serpentine, smectite & green-Hydra-red-ortho \\
0.5 micron & ilmenite, magnetite & NUV-blue-green-orange \\
\hline
\end{tabular}

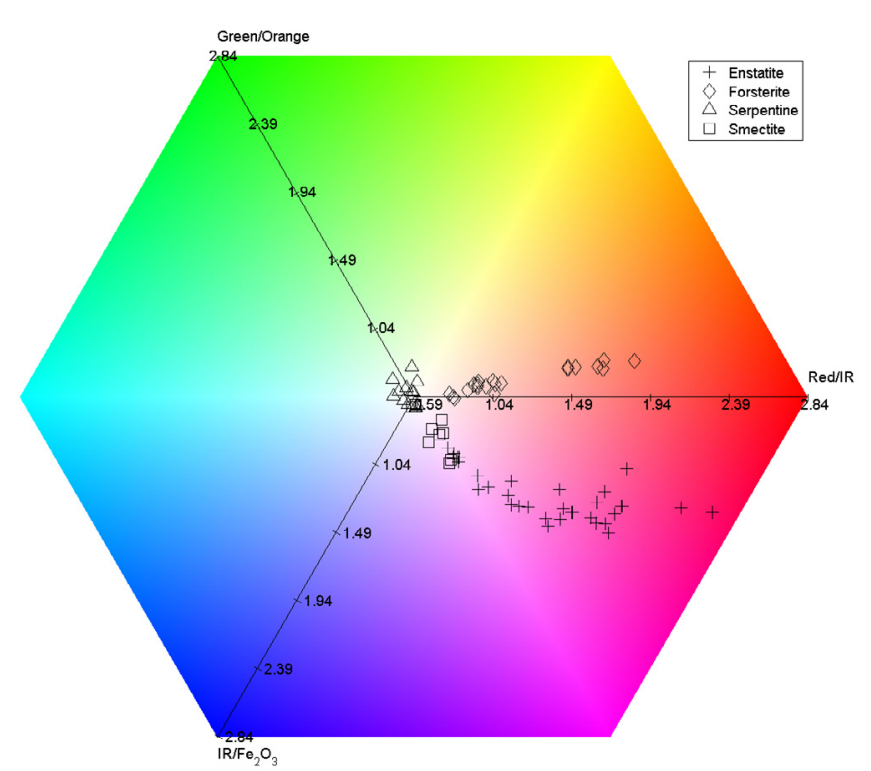

Fig. 10. Filter ratios and RGB color map.

The surface of comet 67P is mapped for the time between July-December 2014 in resolutions down to $15 \mathrm{~cm} / \mathrm{px}$ with various NAC filter combinations. The comet surface shows variations in reflectance of about 20\% (Sierks et al. 2015). Figure 11 shows a composite image of comet 67P in the suggested RGB ratios. The color image is overlaid on a grayscale image to display the surface features together with the surface colors. This image shows the neck region together with the two lobes of comet 67P. The overall comet does not display a main color difference, but local color variations are visible especially in the bright Hapi region, inside the pits, and in the depressions (Sierks et al. 2015; Fornasier et al. 2015). In the RGB colors defined by us, these regions of comet $67 \mathrm{P}$ are orange, while the rest of the surface of both lobes is blueish. In these RGB colors, orange indicates olivine-rich regions, while blueish would indicate pyroxene-rich regions. To draw a reliable conclusion, a detailed analysis of the comet surface needs to be performed on photometrically corrected coma-contribution-removed images, and the resulting spectra obtained from NAC images need to be investigated.

The observations taken from various phase angles were the basis for a study of the spectrophotometric properties of the 67P nucleus by Fornasier et al. (2015) and the global spectrum of the comet together with its head, neck, and body are investigated from the spectrum generated from OSIRIS observations. The surface spectral features are not resolved in the global spectrum, except for an excess in the $700-750 \mathrm{~nm}$ region. With the photometric corrections of Fornasier et al. (2015), the high-resolution surface data will be photometrically corrected. The methods presented in this study will be applied to these data for a detailed analysis of the comet regions and surface structures. 


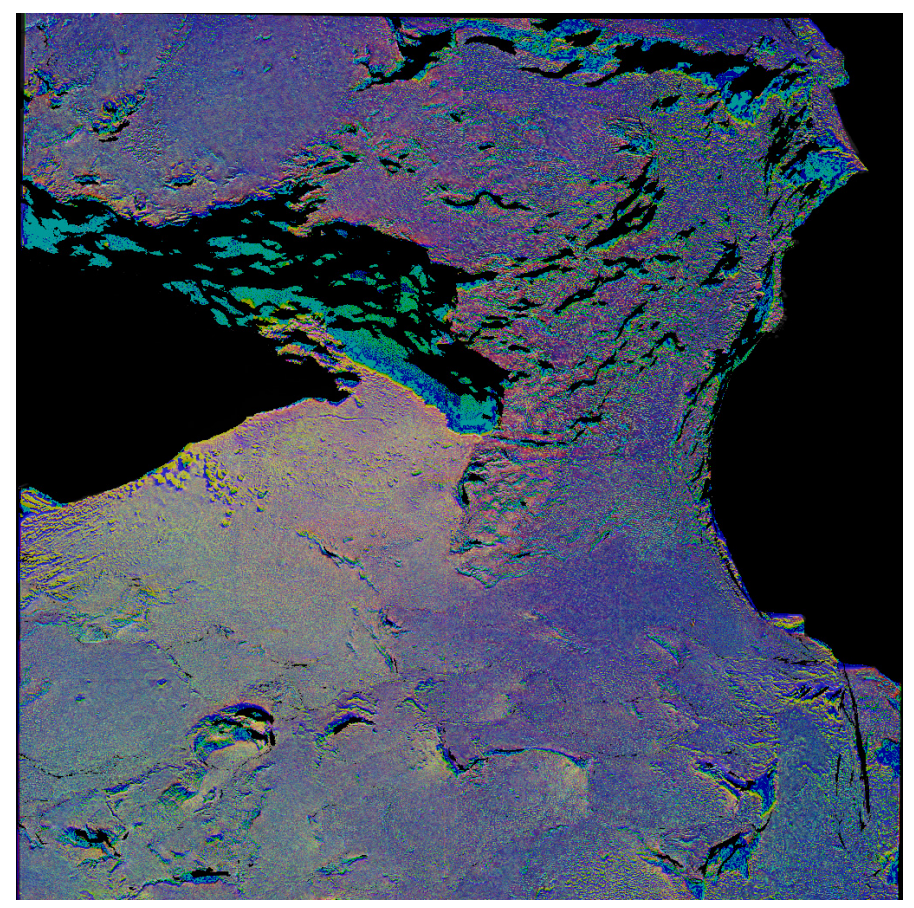

Fig. 11. Comet $67 \mathrm{P}$ in proposed $\mathrm{RGB}$ colors $(\mathrm{R}=\mathrm{red} / \mathrm{IR}, \mathrm{G}=$ green/orange, $\mathrm{B}=\mathrm{IR} / \mathrm{Fe}_{2} \mathrm{O}_{3}$ ) overlaid on a grayscale image for distinction. The color sequence was taken on 22/08/2014 around 01:42 UT. The resolution is $1.3 \mathrm{~m} / \mathrm{px}$, and the distance to the comet is $70 \mathrm{~km}$.

Acknowledgements. OSIRIS was built by a consortium of the Max-PlanckInstitut für Sonnensystemforschung, Göttingen, Germany, the CISAS University of Padova, Italy, the Laboratoire d'Astrophysique de Marseille, France, the Instituto de Astrofísica de Andalucia, CSIC, Granada, Spain,the Research and Scientific Support Department of the European Space Agency, Noordwijk, The Netherlands, the Instituto Nacionalde Técnica Aeroespacial, Madrid, Spain, the Universidad Politéchnica de Madrid, Spain, the Department of Physics and Astronomy of Uppsala University, Sweden, and the Institut für Datentechnik und Kommunikationsnetze der Technischen Universität Braunschweig, Germany. The support of the national funding agencies of Germany (DLR), France (CNES), Italy (ASI), Spain (MEC), Sweden (SNSB), and the ESA Technical Directorate are gratefully acknowledged. The OSIRIS filters flown on Rosetta/OSIRIS were manufactured by Spectrogon AB, based in
Täby, Sweden. We thank Michael J. Gaffey for his comments on the manuscript and the suggestions for the improvement of the presented work. We thank Lucille Le Corre for the discussions during this work. We are grateful to Martin Hilchenbach and Cecile Engrand from the COSIMA team for providing their calibration minerals list. We are thankful to Edward A. Cloutis for sharing his mixture spectra and Guneshwar Singh Thangjam for his collaboration. We thank Sihane Merouane for the discussions during the review process. This research utilizes spectra from NASA RELAB facility at Brown University. This research has made use data from HOSERLab, which was established by the University of Winnipeg, the Canadian Space Agency (CSA), and the Canada Foundation for Innovation and the Manitoba Research Innovations Fund. The laboratory spectral data acquisition and analysis is supported by CSA and the Natural Science and Engineering Research Council of Canada. This research has made use of NASA's Astrophysics Data System Bibliographic Services.

\section{References}

Capaccioni, F., Coradini, A., Filacchione, G., et al. 2015, Science, 347, aaa0628 Combi, M. R., Tenishev, V. M., Rubin, M., Fougere, N., \& Gombosi, T. I. 2012, ApJ, 749, 29

Fornasier, S., Hasselmann, P. H., Barucci, M. A., et al. 2015, A\&A, 583, A30

Gaffey, S. J. 1986, Am. Mineral., 71, 151

Hanner, M. S. 2003, in Astromineralogy, ed. T. K. Henning (Berlin: Springer Verlag), Lect. Notes Phys., 609, 171

Hanner, M. S., \& Zolensky, M. E. 2010, in Lect. Notes Phys. 815, ed. T. Henning (Berlin: Springer Verlag), 203

Henning, T. 2010, Astromineralogy (Berlin: Springer Verlag), Lect. Notes Phys., 815

Keller, H. U., Barbieri, C., Lamy, P., et al. 2007, Space Sci. Rev., 128, 433

Langevin, Y., Kissel, J., Bertaux, J.-L., \& Chassefiere, E. 1987, A\&A, 187, 761

Le Corre, L., Reddy, V., Nathues, A., \& Cloutis, E. A. 2011, Icarus, 216, 376

Li, J.-Y., Besse, S., A'Hearn, M. F., et al. 2013, Icarus, 222, 559

Lisse, C. M., VanCleve, J., Adams, A. C., et al. 2006, Science, 313, 635

Pommerol, A., Thomas, N., El-Maarry, M. R., et al. 2015, A\&A, 583, A25

Reddy, V., Nathues, A., Le Corre, L., et al. 2012, Science, 336, 700

Rivkin, A. S., Howell, E. S., Vilas, F., \& Lebofsky, L. A. 2002, Asteroids III, 235

Sierks, H., Barbieri, C., Lamy, P., et al. 2015, Science, 347, aaa1044

Sitko, M. L., Lisse, C. M., Kelley, M. S., et al. 2011, AJ, 142, 80

Snodgrass, C., Tubiana, C., Bramich, D. M., et al. 2013, A\&A, 557, A33

Sunshine, J. M., A'Hearn, M. F., Groussin, O., et al. 2006, Science, 311, 1453

Thomas, N., Sierks, H., Barbieri, C., et al. 2015, Science, 347, aaa0440

Vilas, F. 1994, Icarus, 111, 456

Weidenschilling, S. J. 2004, in Comets II (Tucson: University of Arizona Press), 97

Wooden, D. H., Harker, D. E., Woodward, C. E., et al. 1999, ApJ, 517, 1034

Zolensky, M. E., Zega, T. J., Yano, H., et al. 2006, Science, 314, 1735

Pages 11 to 12 are available in the electronic edition of the journal at http://www . aanda. org 


\section{Appendix A: Additional figures}
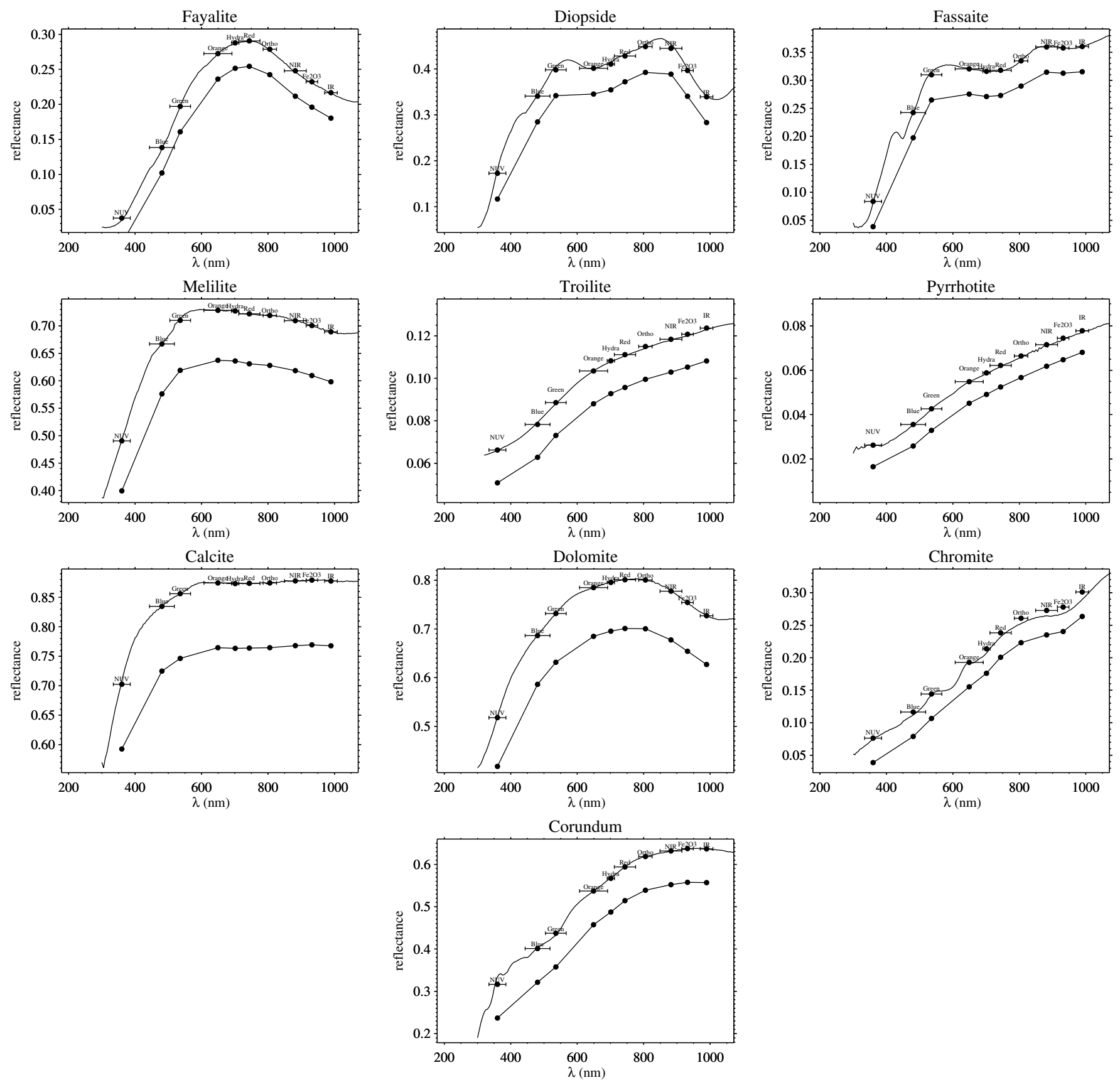

Fig. A.1. Absolute spectra of anhydrous silicates, hydrated silicates, sulfides, carbonates, and oxides as they would be observed through the full set of OSIRIS NAC filters. The solid lines above are laboratory data of the corresponding mineral, with the central wavelengths of NAC (dots) and with their bandwidths (horizontal bars), while resampled laboratory data are arbitrarily (lowered by 1/8 of the maxima) offset for display. 


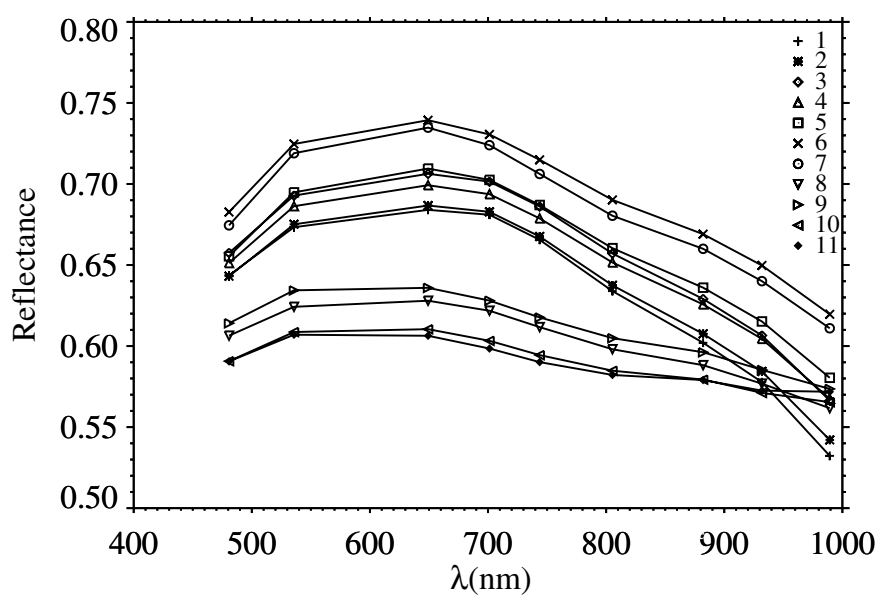

Fig. A.2. Absolute spectrum of the mixtures listed in Table 3 as seen by OSIRIS NAC filters.
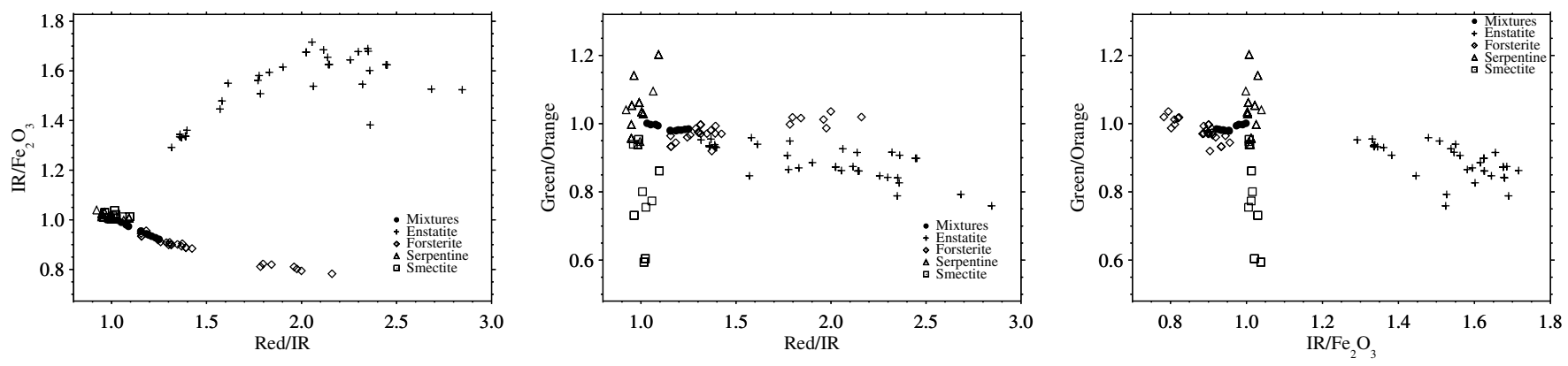

Fig. A.3. Defined ratios for RGB images together with mixtures.

\section{Appendix B: RELAB data}

Table B.1. References for RELAB data.

\begin{tabular}{|c|c|}
\hline Mineral & RELAB file \\
\hline Enstatite & $\begin{array}{l}\text { C2PE13, BKR1PE011, C2PE12, C2PE14, C2PE15, C2PE19, CAPE22, SAPE22, C1PE24, C2PE25, S2PE25, } \\
\text { C1PE30, C4PE30, C5PE30, C6PE30, C1PE31, C3PE31, C1PE32, C2PE32, C5PE32, C3PE34, CAPE34, CBPE34, } \\
\text { CBPE34, CCPE34, CDPE34, CEPE34, CFPE34, CHPE34, CIPE34, CJPE34, C1PP13, C1PP43, C1PP87 }\end{array}$ \\
\hline Forsterite & $\begin{array}{l}\text { C1PO76, C1PO77, CAPO04, C1PO50, CAPO50, C3PO51, C1PO52, C3PO53, C3PO54, LAPO54, C1PO60, C3PO61, } \\
\text { C1PO40, c1ol02, c2ol02, c10103, c2ol03, c1ol04, c2ol04, c1ol10, c10113, c10113 }\end{array}$ \\
\hline Serpentine & C1FB19, C2SM80, C1SR91, CASR14, CASR15, CASR16, CASR19, CASR20, CASR22, CASR24, C1SR64, C1SR64A \\
\hline Smectite & CCC04A, CCC04B, CCC04C, CJB170, CJB173, CJB173, CJB176, CJB177, CJS177, CJS178 \\
\hline Diopside & $\begin{array}{l}\text { BKR1DD074, C1PP20, C1PP21, C4PP21, C5PP21, NBPP21, C1PP22, C3PP22, } \\
\text { C1PP23, C5PP23, NBPP23, C1PP25, C1PP26 }\end{array}$ \\
\hline Fayalite & $\begin{array}{l}\text { C1DD46, C1DD98, C1DD98P, C1PF30, C1PO70, C1PO71, C1PO72, C1PO73, C1PO74, C1PO75, } \\
\text { C1PO78, C1PO79, CAPO05, C1PO58, C3PO59 }\end{array}$ \\
\hline Melilite & CAME04, CAME05, CAME06, CAME07, CAME08, C1SC66, CASC66, C1SC67, CASC67 \\
\hline Fassaite & C1PP103A, C1PP91, C1SC69, C1SC70 \\
\hline Calcite & $\begin{array}{l}\text { CACA10, CACB10, CACB11, CACB12, CBCB12, C1CY05, C1GR01, C1MA44, C1OS19, C1OS20, } \\
\text { C1PC50, C2PC50, C1SG09, C2SG55, C1SG67, C1SS11, C2XT10, C1XT10 }\end{array}$ \\
\hline Dolomite & $\begin{array}{l}\text { CACB03, CBCB03, CACB17, C1CY07, C1JB779, C2PD05, C1SH07, C1SH08, C2SH08, C1SH78, } \\
\text { C1SH79, C1SH85, C1SH86, C1SH87, C1SH90, C1SH91 }\end{array}$ \\
\hline Pyrrhotite & C1PY01, C2PY01, C1RP02, C1SC76 \\
\hline Troilite & $\begin{array}{l}\text { CAEA01, CBEA01, CAEA09, CBEA09, C1MB06, CAMB06, CBMB06, ССMB06, CDMB06, CEMB06, } \\
\text { CFMB06, C1SC64, C1TB05, C1TB06, C1TB49, S1TB49, C1TB50 }\end{array}$ \\
\hline Chromite & $\begin{array}{l}\text { CACR11, CACR12, CACR13, CACR14, CACR15, CACR16, CACR17, CACR18, CACR19, C1PC21, S1PC21, } \\
\text { C1PC22, CATW01 }\end{array}$ \\
\hline Corundum & CACD01 \\
\hline Magnetite & C1JB764, CAMG04, CAMG05, CAMG07, CAMG11, CAMG21 \\
\hline Ilmenite & $\begin{array}{l}\text { C1PP26, C1LR182, CAMR05, CBMR05, CDMR05, CEMR05, CCMR05, C1MR06, C1MR07, C1MR08, C1MR09, } \\
\text { C1MR126, C1MR127, C1MR128, C1MR129, C2PI03, C1PI06, CAPI06, C4PI06A, C1PI06A, CBPI06, C2PI06, } \\
\text { CPI06B, CAPI07, CARS69, CBRS69, CCRS69, CDRS69, CERS69, C4RS86, C6RS86, C1SR58, C1SR58A }\end{array}$ \\
\hline
\end{tabular}

